\title{
The methyl-CpG-binding protein CIBZ suppresses myogenic differentiation by directly inhibiting myogenin expression
}

\author{
Yu Oikawa ${ }^{1,2}$, Reiko Omori ${ }^{1}$, Tomonori Nishii ${ }^{1}$, Yasumasa Ishida ${ }^{1}$, Masashi Kawaichi ${ }^{1}$, Eishou Matsuda ${ }^{1}$

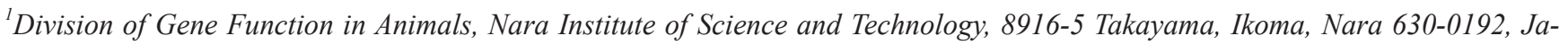 \\ pan; ${ }^{2}$ Department of Pathology and Applied Neurobiology, Graduate School of Medical Sciences, Kyoto Prefectural University of \\ Medicine, Kawaramachi Hirokoji Kajii-cho, Kamigyo-ku, Kyoto 602-8566, Japan
}

Postnatal growth and regeneration of skeletal muscle are carried out mainly by satellite cells, which, upon stimulation, begin to express myogenin (Myog), the critical determinant of myogenic differentiation. DNA methylation status has been associated with the expression of $M y o g$, but the causative mechanism remains almost unknown. Here, we report that the level of CIBZ, a methyl-CpG-binding protein, decreases upon myogenic differentiation of satellitederived $\mathrm{C} 2 \mathrm{C} 12$ cells, and during skeletal muscle regeneration in mice. We present data showing that the loss of CIBZ promotes myogenic differentiation, whereas exogenous expression of $C I B Z$ impairs it, in cultured cells. CIBZ binds to a Myog promoter-proximal region and inhibits Myog transcription in a methylation-dependent manner. These data suggest that the suppression of myogenic differentiation by CIBZ is dependent, at least in part, on the regulation of Myog. Our data show that the methylation status of this proximal Myog promoter inversely correlates with Myog transcription in cells and tissues, and during postnatal growth of skeletal muscle. Notably, induction of Myog transcription by CIBZ suppression is independent of the demethylation of CpG sites in the Myog promoter. These observations provide the first reported molecular mechanism illustrating how Myog transcription is coordinately regulated by a methyl-CpG-binding protein and the methylation status of the proximal Myog promoter.

Keywords: CIBZ; MBP; myogenin; muscle differentiation; regeneration

Cell Research (2011) 21:1578-1590. doi:10.1038/cr.2011.90; published online 31 May 2011

\section{Introduction}

In rodents, skeletal muscles grow dramatically from birth to adulthood. Adult skeletal muscle is capable of repeated self-repair, throughout life, in response to damage caused by injury or myopathies [1,2]. Such postnatal development and self-repair of skeletal muscles is mainly attributable to muscle progenitor cells called satellite cells, which are located between the basal lamina and the plasma membrane of muscle fibers $[1,3]$. Satellite cells account for about $30 \%$ of the muscle nuclei in neonatal mice, but decline to $2-6 \%$ in 2 -month-old adults [3]. As

Correspondence: Eishou Matsuda

Tel: +81-743-72-5537; Fax: +81-743-72-5539

E-mail: ematsuda@bs.naist.jp

Received 26 July 2010; revised 20 January 2011; accepted 10 February 2011; published online 31 May 2011 adulthood is reached, the residual satellite cells become quiescent, but upon activation they divide to repopulate the satellite cell pool and to generate a large number of daughter myoblasts. These daughter myoblasts subsequently undergo myogenic differentiation, in which they fuse and form multinucleated myotubes [1,4]. Activation of quiescent satellite cells and subsequent myogenic differentiation are controlled by various transcription factors, chief among which are the myogenic regulatory factors (MRFs) Myf5, MyoD, myogenin (Myog) and MRF4 [5]. MyoD and Myf5 are expressed in proliferating and undifferentiated myoblasts, whereas Myog is induced during early differentiation. In agreement with these expression patterns, MyoD and Myf5 establish the myogenic lineage, while Myog directly controls the differentiation of myoblasts $[4,5]$. MRF4, on the other hand, appears to function as a differentiation factor in later fibers. Induction of Myog is essential for the differentiation of 
myoblasts that contributes to the formation of myotubes and fibers: mice deficient for Myog form myoblasts but do not develop mature skeletal muscle [6]. Hence, a deeper understanding of the transcriptional regulation of Myog will provide important insights into the molecular mechanism of myogenic differentiation.

Myog transcription is controlled by a $1.5-\mathrm{kb} 5^{\prime}-$ regulatory region (nucleotides -1565 to +18 ), which is sufficient to recapitulate the major features of Myog expression during embryonic and fetal development [7]. Cumulative evidence indicates that the promoter region $(-184$ to +18$)$ is indispensable for Myog expression [8, 9]. Transcription of Myog is stimulated mainly by MRFs or by members of the myocyte enhancer factor 2 (MEF2) family, through binding to the E-box elements or to the MEF2-binding site of the Myog promoter, respectively $[5,10]$. By contrast, Myog expression is negatively regulated by the inhibitors of DNA-binding (Id) family, which block the stimulatory effect of MRFs by forming inactive heterodimers with them [11]. Myog is also negatively regulated by other transcriptional repressors that bind to the E-box elements, such as MyoR [12]. Whereas considerable progress has been made in elucidating how Myog is regulated through its E-box elements and MEF2binding site, much less is known about the patterns of DNA methylation of this muscle-specific gene.

Cytosine-5 DNA methylation in mammals is essential for important functions such as cell differentiation, imprinting and X-inactivation [13]. Treatment of 10T1/2 fibroblasts with the DNA demethylating reagent 5-aza-dC, or expression of antisense Dnmt1, results in myotube formation, suggesting that global demethylation plays a role in myogenic differentiation $[14,15]$. Similarly, 5-aza-dC treatment induces Myog expression in myoblast cells, implying that DNA methylation is also involved in the suppression of Myog transcription [16, 17]. Since the 51-kb region between Myog and its upstream gene lacks CpG islands, DNA methylation in the vicinity of the promoter is probably responsible for Myog silencing. Consistent with this, Lucarelli et al. reported that the methylation status of a single CCGG site at $340 \mathrm{bp}$ upstream from the transcriptional start site (TSS) affects Myog transcription in mouse tissues and in $\mathrm{C} 2 \mathrm{C} 12$, a skeletal muscle satellite-derived myoblast cell line [17]. This HpaII recognition site (CCGG), which is in the region proximal to the Myog promoter, was identified using the methylationsensitive endonuclease HpaII-PCR assay. However, we currently lack information about which methylated $\mathrm{CpG}$ binding proteins (MBPs) bind to this site, or a detailed $\mathrm{CpG}$ methylation profile of the proximal Myog promoter around the site.

The $\mathrm{C} 2 \mathrm{C} 12$ cell line is a well-established model to investigate the cellular and molecular mechanisms of muscle differentiation [18]. This in vitro system faithfully recapitulates the in vivo differentiation program. When cultured in differentiation medium (DM), C2C12 cells undergo terminal myogenic differentiation. We reported previously that a novel MBP member, CIBZ (ZBTB38 in human) [19], represses the Gal4-driven SV40 promoter [20]; it can bind to methylated $\mathrm{CpG}$ through its zinc fingers (unpublished data). We found that CIBZ is localized in both the nucleus and the cytoplasm of NIH3T3 cells [20] and C2C12 cells (unpublished data); its level is high in $\mathrm{C} 2 \mathrm{C} 12$ cells but decreases upon DM induction [21].

We now show that CIBZ is down-regulated during skeletal muscle regeneration, and that it suppresses C2C12 myoblast differentiation. Our data reveal that CIBZ's role in myogenic differentiation is dependent, at least in part, on the negative regulation of its direct target, Myog. CIBZ binds to a 451-bp proximal region of the Myog promoter (Myog451). Using a combination of approaches, we show that CIBZ binds directly to and represses the activity of Myog451 in a methylationdependent manner. We provide evidence that the methylation status of Myog451correlates with Myog transcriptional activation in cells and tissues, and during postnatal growth of skeletal muscle. These findings suggest a viable therapeutic approach for the treatment of skeletal muscle disorders.

\section{Results}

CIBZ expression is down-regulated during myoblast differentiation and skeletal muscle regeneration

We sought to determine the role of CIBZ in myogenic differentiation. Expression of Myog and myosin heavy chain (MHC), markers of early and late stages of myogenic differentiation, respectively, was monitored to assess the cells' differentiation state [22]. As seen in Figure 1A, RT-PCR and immunoblot analysis showed that induction of $\mathrm{C} 2 \mathrm{C} 12$ cells in DM resulted in early differentiation within 8-24 h, and in late differentiation after $48 \mathrm{~h}$. CIBZ protein declined quickly within $8 \mathrm{~h}$ and was barely detectable at 24-72 h during differentiation, whereas $C I B Z$ mRNA levels remained relatively stable.

In response to muscle injury in which myofibers are damaged, satellite cells begin to expand and differentiate to replace the lost myofibers. To investigate the expression of CIBZ protein during muscle regeneration in mice, cardiotoxin (CTX) was used. Treatment of the tibialis anterior muscle with CTX is a common and reproducible method to induce myonecrosis and subsequent muscle regeneration while leaving the innervating nerve intact $[2$, 23]. As shown in Figure 1B, treatment with CTX in skel- 
etal muscle induced regeneration on day 3, evidenced by the up-regulation of Myog at both the mRNA and protein levels. These observations confirm previous reports [24, 25]. Conversely, CIBZ protein was down-regulated on days 1 and 3 post-injection, whereas $C I B Z$ mRNA levels did not change appreciably.

As the down-regulation of CIBZ occurred before the up-regulation of Myog (Figure 1A and 1B) in these experiments, abrogation of CIBZ may be required for myogenic differentiation in $\mathrm{C} 2 \mathrm{C} 12$ myoblasts, and for skeletal muscle regeneration.

\section{CIBZ regulates myogenic differentiation in $\mathrm{C} 2 \mathrm{C} 12$ cells}

To clarify the role of CIBZ in myogenic differentiation, we first examined the effect of its depletion by siRNA. Inhibition of CIBZ 24-48 h after single siRNA transfection in $\mathrm{C} 2 \mathrm{C} 12$ myoblasts induced Myog, but not MHC (data not shown). Since this period may be insufficient to yield an observable phenotype, we used repeated transfection of siRNA, to sustain a long-term knockdown effect. siRNA-mediated knockdown of CIBZ on day 5 , three days after a second transfection, resulted in induction of the MHC protein (Figure 2A), although
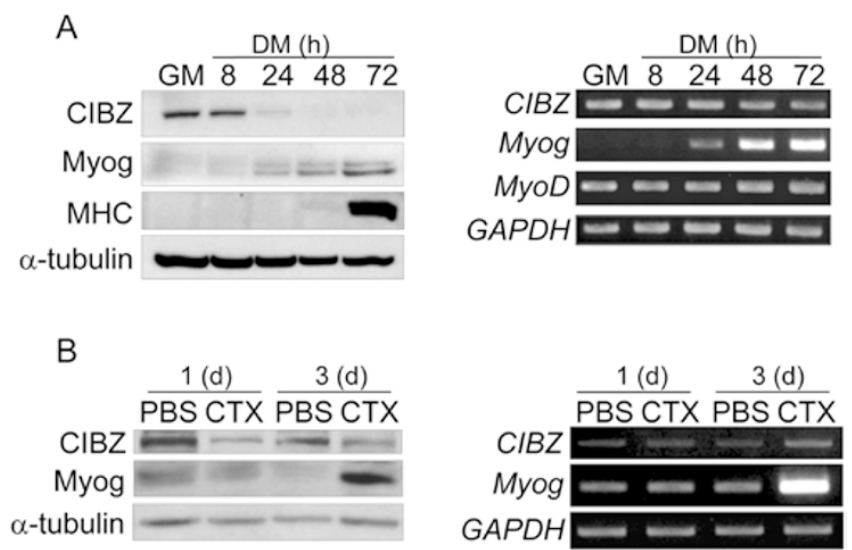

Figure $1 \mathrm{CIBZ}$ is down-regulated during myogenic differentiation in $\mathrm{C} 2 \mathrm{C} 12$ cells, and in skeletal muscle regeneration. (A) DM was used to induce myogenic differentiation. Total cell lysates were collected at the indicated time points, and analyzed by western blotting (left panels) and semiquantitative RT-PCR (right panels). GM: growth medium. The indicated proteins were detected by their corresponding antibodies. $\alpha$-tubulin and the glyceraldehyde-3-phosphate dehydrogenase $(G A P D H)$ gene were used as controls for western blots and RT-PCR, respectively. (B) Tibialis anterior muscles from 6-week-old C57BL/6 mice, 1 and 3 days after CTX-induced injury or PBS control injection, were dissected, and whole-cell lysates were prepared and analyzed by western blotting (left panels) and semiquantitative RT-PCR (right panels). Results shown are representative of three independent experiments. the efficiency was much lower than that attained by DM treatment. These data indicate that CIBZ abrogation is capable, at least to some extent, of inducing myogenic differentiation.

To examine whether CIBZ silencing accelerates DMinduced multinucleated myotube formation, we knocked down the expression of CIBZ by siRNA in $\mathrm{C} 2 \mathrm{C} 12$ cells for 1 day, and then cultured the cells in DM. Immunoblot analysis showed that $C I B Z$ siRNA, but not the control siRNA, enhanced the up-regulation of MHC induced by culturing in DM, in a time-dependent manner (Supplementary information, Figure S1A). CIBZ inhibition resulted in a remarkable enhancement of myotube formation and up-regulation of MHC on days 2 (Supplementary information, Figure S1B-S1D) and 3 (Figure 2B and 2C) after switching to DM.

To explore whether ectopically expressed CIBZ has a reciprocal effect on myogenic differentiation, we transfected Myc-tagged CIBZ into C2C12 cells for 1 day (Figure 2D, left panel), and then treated the cells with DM for 2 days. As anticipated, Myc-CIBZ significantly reduced the DM-induced up-regulation of Myog and MHC protein levels (Figure 2D, right panel), indicating that ectopic CIBZ expression impedes myogenic differentiation.

CIBZ's role in myogenic differentiation is at least partially dependent on Myog gene regulation

To investigate the molecular mechanism underlying CIBZ-regulated myogenic differentiation, we examined whether CIBZ abrogation affects the transcription of MRFs. RT-PCR analysis showed that CIBZ depletion activates the expression of only Myog among the MRF genes; MyoD, Myf5 and MRF4 transcription was unaffected (Figure 3A). Immunoblot analysis revealed that the inhibition of CIBZ expression by siRNA-1 and -2 resulted in a significant up-regulation of Myog protein, but not of MyoD. These data suggest that CIBZ acts as a negative regulator of the Myog gene.

Next, we examined whether overexpression of CIBZ (Supplementary information, Figure S2) inhibits Myog. As shown in Figure 3B, overexpression of CIBZ in $\mathrm{C} 2 \mathrm{C} 12$ cells suppressed the up-regulation of Myog at both the mRNA and protein levels under DM induction. Considering that CIBZ is an MBP [19], we next asked whether the CIBZ zinc finger 1-5 (ZF1-5) region, which can bind independently to methylated $\mathrm{CpG}$ (Figure 5B), was required for the suppression of Myog transcription. As seen in Figure 3B, the CIBZ $\triangle \mathrm{ZF} 1-5$ mutant (lacking the ZF1-5 domain) failed to repress $M y o g$ expression. This result reveals that the suppression of Myog gene by CIBZ may be DNA methylation-dependent, and, in 
A

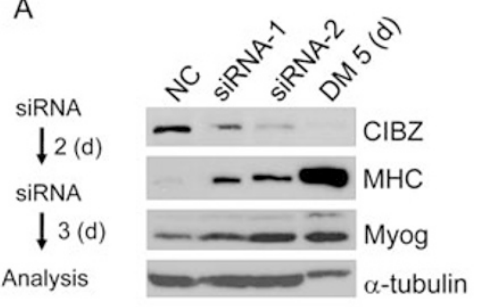

C

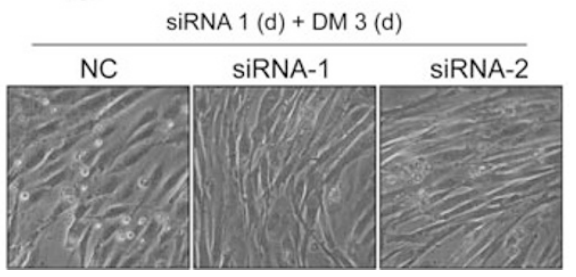

B
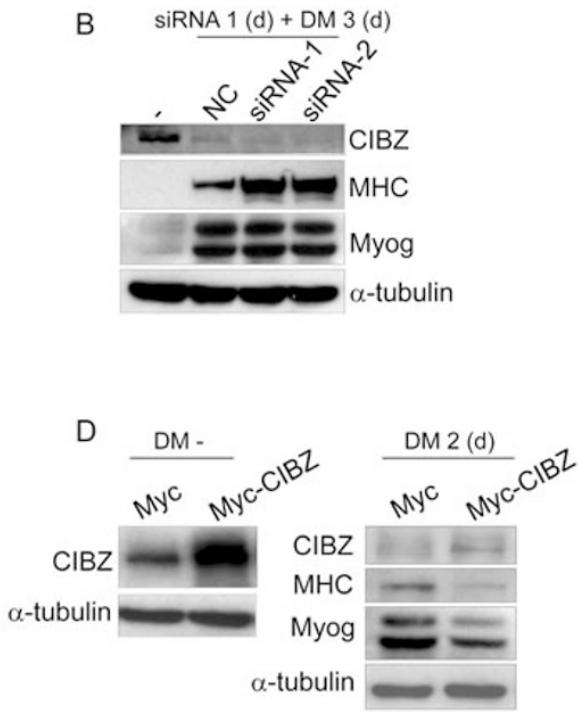

Figure 2 CIBZ negatively regulates terminal differentiation of C2C12 myoblasts. (A) Repeated knockdown of CIBZ up-regulates the expression of $\mathrm{MHC}$ in $\mathrm{C} 2 \mathrm{C} 12$ myoblasts. $\mathrm{C} 2 \mathrm{C} 12$ cells were transfected with two CIBZ siRNAs or a scrambled siRNA negative control $(\mathrm{NC})$. After the first transfection, the cells were transfected again with siRNA on day 2 . Total cell lysates were isolated on day 5 , and subjected to immunoblot analysis with the indicated antibodies. $\alpha$-Tubulin was used as a loading control. (B, C) C2C12 cells were transfected with CIBZ siRNA or NC for 1 day, and then cultured in DM for an additional 3 days. (B) Total cell lysates were subjected to immunoblot analysis with the indicated antibodies. Untransfected $\mathrm{C} 2 \mathrm{C} 12$ cells were also used as a control (lane 1, "-"). (C) CIBZ siRNA-treated C2C12 cells were photographed under a phase-contrast microscope. (D) Exogenous CIBZ expression in $\mathrm{C} 2 \mathrm{C} 12$ cells impedes myogenic differentiation induced by DM. C2C12 cells were transiently transfected with Myc or Myc-CIBZ for 1 day, and then treated with DM for 2 days. Total cell lysates were subjected to immunoblot analysis. Results shown are representative of three independent experiments.
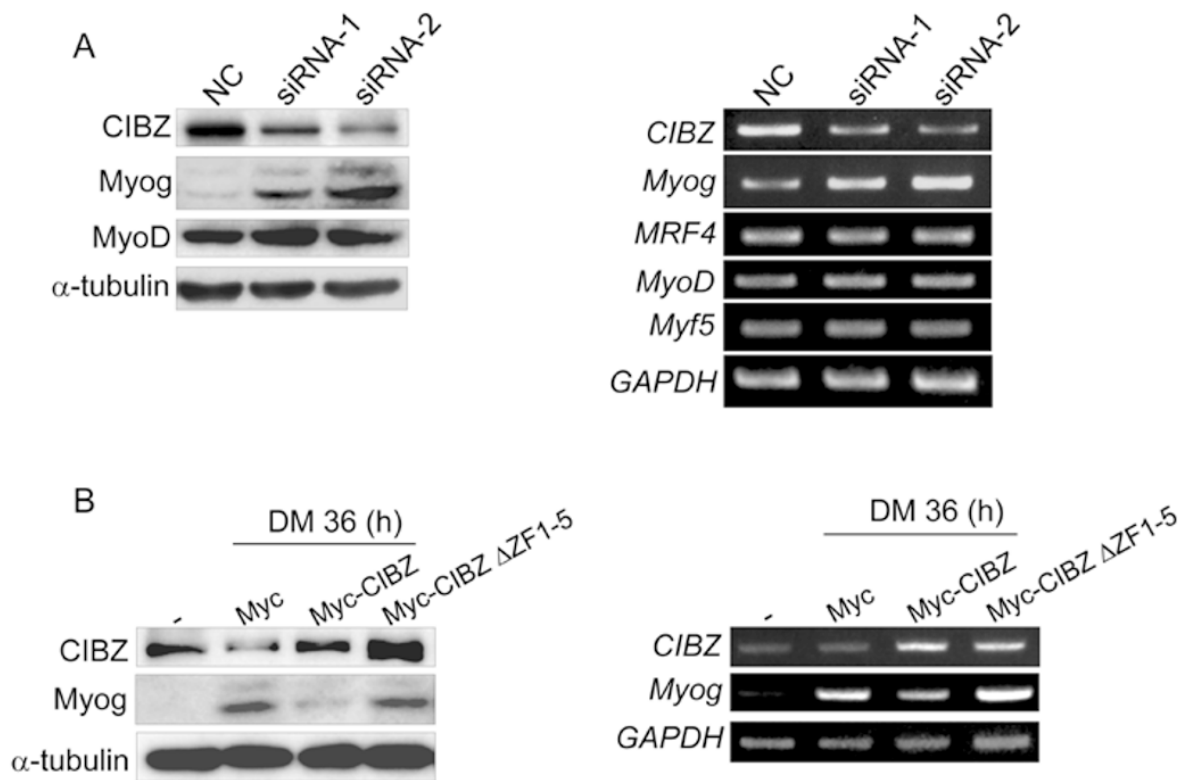

Figure 3 CIBZ protein level regulates the expression of Myog in $\mathrm{C} 2 \mathrm{C} 12$ myoblasts. (A) At $36 \mathrm{~h}$ after transfection with two CIBZ siRNAs or NC, expression of the indicated proteins and genes was detected by western blotting (left panels) and semiquantitative RT-PCR (right panels), respectively. $\alpha$-tubulin and GAPDH served as loading controls for immunoblotting and RT$\mathrm{PCR}$, respectively. (B) C2C12 cells were transfected with Myc, Myc-CIBZ and Myc-CIBZ $\triangle \mathrm{ZF} 1-5$ for $24 \mathrm{~h}$, treated with DM for $36 \mathrm{~h}$, and then cell lysates were analyzed by western blotting (left panels) or by semiquantitative RT-PCR (right panels). 
conjunction with the CIBZ loss-of-function data (Figure 3A), suggests that CIBZ's role in differentiation is dependent, at least in part, on the negative regulation of Myog transcription.

CIBZ binds to the Myog promoter in a DNA methylationdependent manner in C2C12 cells

We next used a chromatin immunoprecipitation (ChIP) assay to investigate whether CIBZ binds to the Myog promoter in proliferating $\mathrm{C} 2 \mathrm{C} 12$ cells. Chromatin from $\mathrm{C} 2 \mathrm{C} 12$ cells was prepared and immunoprecipitated with anti-MyoD antibody, as a positive control, normal rabbit $\mathrm{IgG}$, as a negative control, and a new anti-CIBZ antibody (see Methods) which is able to immunoprecipitate endogenous CIBZ in C2C12 cells (data not shown). PCR primers for the ChIP assay were designed to amplify the promoter region $(-185$ to -1$)$ or a more distally located sequence $(-1565$ to -1447$)$ in the 5 '-regulatory region. As expected, MyoD bound to the promoter region containing its binding sites $[9,26]$, but not to the distal sequence, which lacks a binding site (Figure 4A and 4B). Similarly, strong binding of CIBZ was observed at the promoter region but not at the distal region.
To determine whether this binding of CIBZ is dependent on the methylation status of Myog, we treated proliferating $\mathrm{C} 2 \mathrm{C} 12$ cells with $1 \mu \mathrm{M} 5$-aza-dC for 3 days, conditions that are sufficient for almost complete demethylation of the proximal Myog promoter (see Figure 7A, Supplementary information, Figure S5A). Immunoblot data showed that the expression of either CIBZ or MyoD was not greatly changed after the 5-aza-dC treatment (Supplementary information, Figure S3). ChIP analysis revealed that 5 -aza- $\mathrm{dC}$ treatment almost abolished the binding of CIBZ to the Myog promoter region, but did not weaken the binding of MyoD, which binds to CpG-free E-box motifs at the Myog promoter [9], to this region (Figure $4 \mathrm{C}$ ). These results strongly suggest that binding of CIBZ to the Myog promoter is methylationdependent.

CIBZ binds directly to the proximal Myog promoter in a methylation-dependent manner

To obtain direct evidence that CIBZ binds specifically to the Myog promoter in vitro, we conducted gel mobility shift assays. The methyl-CpG-binding motif of CIBZ, ZF1-5, was fused to glutathione S-transferase
A

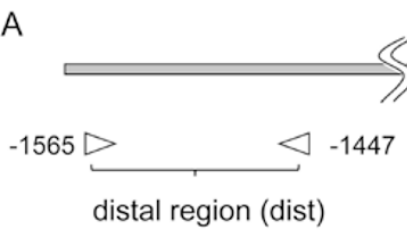

B

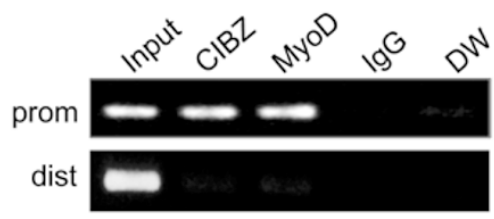

C

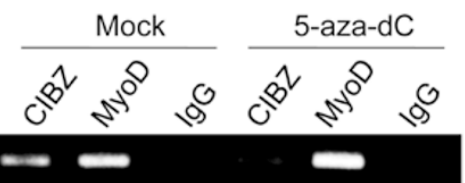

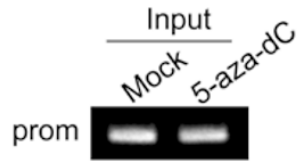

Figure 4 Methylation-sensitive binding of CIBZ to the 5'-regulatory region of Myog. (A) Schematic structure of the 5'-regulatory region of the mouse Myog gene, which contains the amplified promoter fragment $(-185$ to -1$)$ sequence. A MEF2-binding site $(\mathrm{M})$ and two E-boxes $(\mathrm{E})$ located in the promoter are indicated. The TSS (+1) is marked by a bent arrow. Locations of ChIP primers for the promoter region (prom, black arrowheads) and the distal region (dist, empty arrowheads) are shown. (B) ChIP assays were performed with antibodies to CIBZ. Distilled water and non-specific IgG, and an antibody against MyoD, were used as negative controls and as a positive control, respectively. The input DNA (2.5\%), IgG-precipitated DNA and DNA immunoprecipitated with the corresponding antibodies were amplified with primers specific for the promoter region (prom) or distal region (dist) as indicated. (C) ChIP assays with CIBZ and MyoD antibodies in C2C12 cells treated with $1 \mu \mathrm{M}$ 5-aza-dC or mock (DMSO)-treated for 3 days. A promoter region-specific primer (prom) was used to amplify the input and immunoprecipitated DNA. Results shown are representative of four independent experiments. 
A

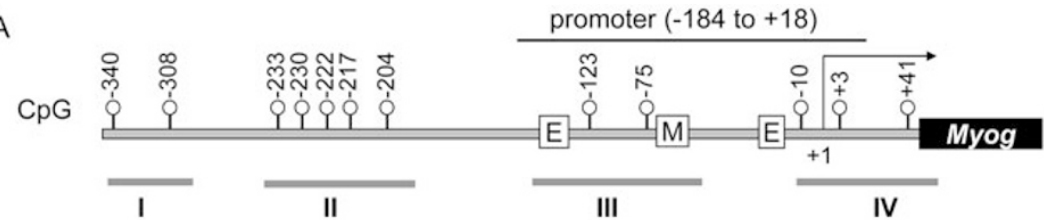

B
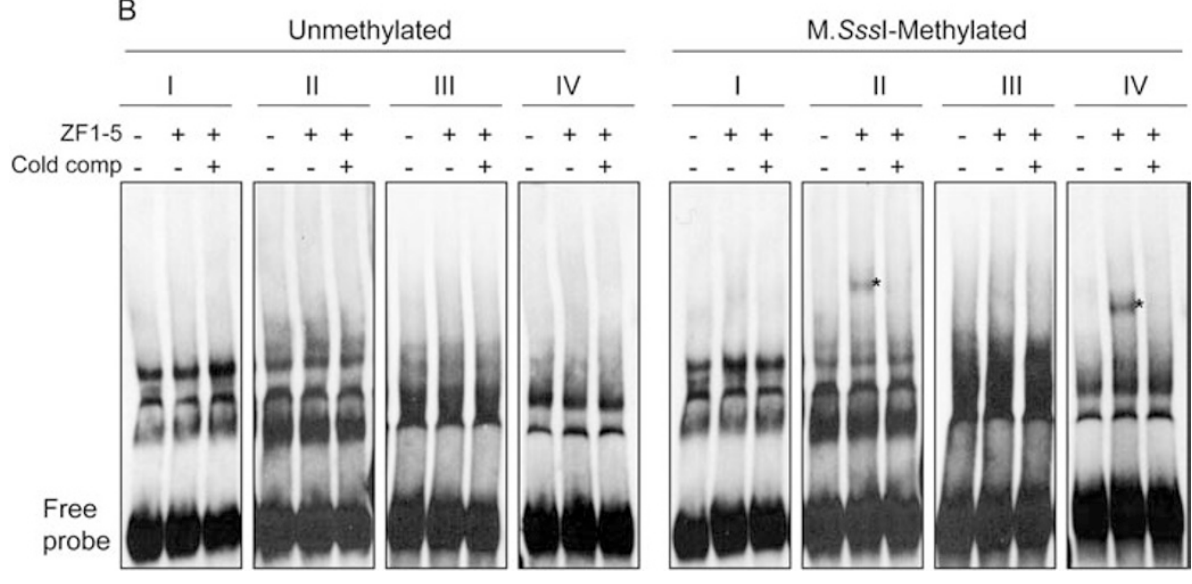

Figure 5 Electrophoretic mobility shift assay (EMSA) of CIBZ binding to the fully methylated proximal Myog promoter. (A) Schematic map showing the locations of the $12 \mathrm{CpG}$ sites (open circles) in the proximal Myog promoter. The sequence locations of four sets of oligonucleotide probes (I to IV) covering the $12 \mathrm{CpG}$ sites are indicated. The TSS is indicated by a bent arrow. (B) EMSA was performed using GST-tagged ZF1-5 of CIBZ with either unmethylated control (left panels) or M.Ssslmethylated probes (right panels). Excess (150-fold) amounts of the corresponding unlabeled probes were used as specific cold competitors (Cold comp). Asterisks represent methyl-CpG-binding complexes. Results shown are representative of three independent experiments.

(GST-ZF1-5) and purified as described previously [21]. To determine whether CIBZ also binds to sites besides those within the Myog promoter (four CpG sites), eight additional $\mathrm{CpG}$ sites were examined, including the -340 $\mathrm{CpG}$ site and a CpG site located at +41 (Figure 5A). Four DNA probes (I, II, III and IV), which harbor 2, 5, 2 and $3 \mathrm{CpG}$ sites, respectively, were designed and methylated in vitro by $\mathrm{CpG}$ methyltransferase $S s s \mathrm{I}$ (M.SssI) (Figure 5A). Neither GST alone (data not shown) nor GSTZF1-5 (Figure 5B) could bind to any of the unmethylated probes. By contrast, GST-ZF1-5 produced gel-retarded complexes with methylated probes II (five $\mathrm{CpG}$ sites) and IV (three $\mathrm{CpG}$ sites). The corresponding unlabeled cold competitors eliminated the formation of these two CIBZDNA complexes, indicating that the binding of CIBZ to these two probes is specific. These data demonstrate that CIBZ can bind directly and specifically to methylated $\mathrm{CpG}$ dinucleotides both within the Myog promoter and in its vicinity in vitro (Figure $5 \mathrm{~A}$ ).

CIBZ negatively regulates the activity of a 451-bp proximal Myog promoter in a methylation-dependent manner

To elucidate the mechanism underlying transcriptional regulation of Myog by CIBZ, a luciferase assay was performed. A 1609-bp Myog 5'-regulatory fragment containing the promoter region was cloned into the promoterless luciferase reporter pGL3-Basic (pGL3-Myog1609). As shown in Supplementary information, Figure S4, ectopic MyoD [26] activated pGL3-Myog1609 luciferase expression in $293 \mathrm{~T}$ cells, while CIBZ overexpression failed to suppress pGL3-Myog1609, suggesting that the negative regulation of Myog by CIBZ is methylation-dependent.

To determine whether CIBZ indeed modulates Myog transcription in a methylation-dependent manner, we cloned a 451-bp proximal Myog promoter fragment (Myog451: -406 to +45 ) into the pCpGL luciferase vector, which is devoid of $\mathrm{CpG}$ dinucleotides [28]. Myog451 was chosen because it contains not only the promoter fragment to which CIBZ binds preferentially (Figure 4B and $5 \mathrm{~B}$ ), but also nine additional $\mathrm{CpG}$ sites including the -340 HpaII recognition site (Figure 6A). The resultant reporter plasmid pCpGL-Myog451 was methylated in vitro by M.SssI, which methylates all $\mathrm{CpG}$ sites, and methylation of this construct was confirmed using HpaII (Figure 6B). As shown in Figure 6C, the luciferase activity of methylated pCpGL-Myog451 in C2C12 cells 
A

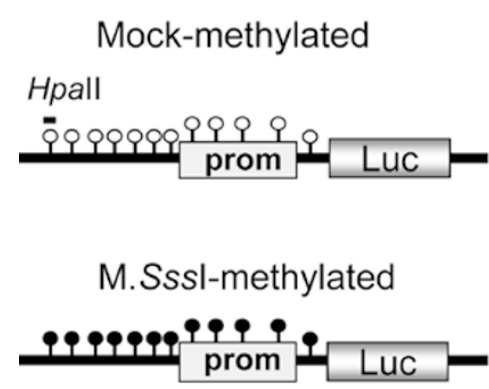

C

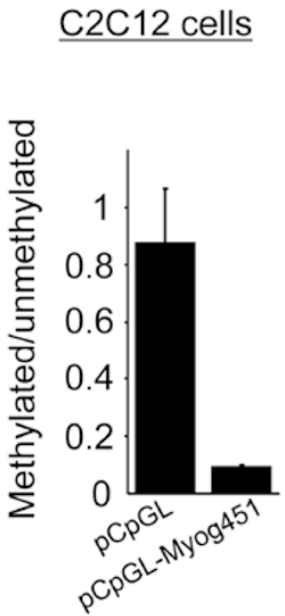

B
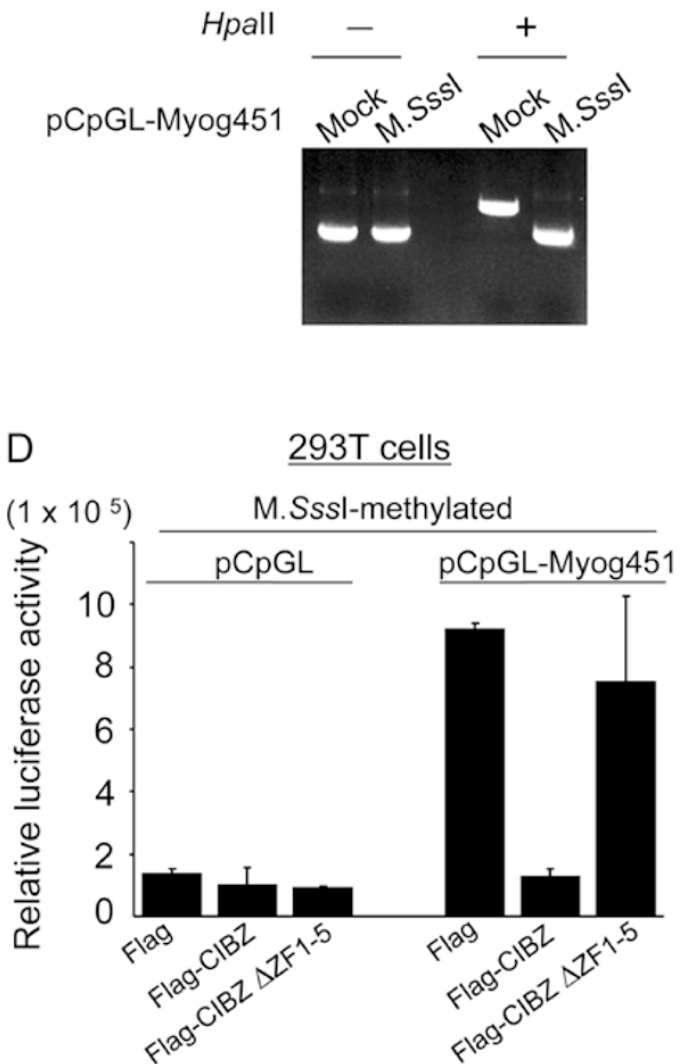

Figure 6 CIBZ negatively regulates the Myog gene in a methylation-dependent manner. (A) Schematic representation of the M.Sssl-methylated pCpGL-Myog451 luciferase reporter construct (lower panel), and the promoter (prom). The mockmethylated control (upper panel) consisted of all components required for in vitro methylation except M.Sssl. Methylated and unmethylated CpGs are marked by filled and open circles, respectively. The Hpall site in Myog451 is indicated in the upper panel. (B) M.Sssl-methylated pCpGL-Myog451 or a mock-methylated control was digested with methylation-sensitive Hpall and analyzed on a $0.8 \%$ agarose gel to verify the efficiency of in vitro methylation. (C) $\mathrm{C} 2 \mathrm{C} 12$ cells were transiently transfected with M.Sssl-methylated or mock-methylated pCpGL and pCpGL-Myog451, together with an internal control pRL-TK, for 2 days. Luciferase activity expressed from M.Sssl-methylated pCpGL and pCpGL-Myog451 was calculated relative to that from unmethylated plasmids, which was normalized to 1. (D) M.Sssl-methylated pCpGL and pCpGL-Myog451 were cotransfected with Flag, Flag-tagged CIBZ and CIBZ $\triangle$ ZF1-5 into 293T cells for 2 days. pRL-TK was cotransfected as an internal control. Relative luciferase activity was determined by normalization to internal control activity. Each bar represents mean \pm standard deviation (SD) derived from three experiments.

was much lower than that of its unmethylated form, indicating that methylation of Myog451 accounts for its transcriptional repression. In 293 T cells, Flag-CIBZ significantly decreased the luciferase activity of methylated pCpGL-Myog451 (Figure 6D and Supplementary information, Figure S4D), whereas CIBZ $\triangle \mathrm{ZF1}$-5 lacked this repressive effect (Figure 6D). We also observed that ectopic expression of CIBZ in 293T cells did not inhibit the luciferase activity of pGL3-Myog451 (data not shown). These data indicate that suppression of Myog promoter activity by CIBZ is methylation-dependent.
Methylation of CpG sites in Myog 451 is inversely correlated with Myog expression

The methylation status of all $12 \mathrm{CpG}$ sites in Myog451 was evaluated by sodium bisulfite sequencing (SBS). As shown in Figure 7A, sequence analysis of individual clones (10 clones per sample) revealed that Myog451 was moderately methylated $(42.5 \%)$ in proliferating $\mathrm{C} 2 \mathrm{C} 12$ cells. Upon DM induction, $\mathrm{CpG}$ methylation of Myog451 decreased slightly (40.0\%) on day 3, but it dropped to $32.5 \%$ on day 5 . Significantly, treatment of C2C12 cells with $1 \mu \mathrm{M} 5$-aza-dC for 3 days led to a dramatic demethylation of Myog451(8.3\%). Notably, CpG 
sites at $-308,-233$ and -230 were more demethylated during myogenic differentiation (Supplementary information, Figure S5B-S5C). In parallel, semiquantitative PCR showed that the expression of Myog was induced by 5 -aza-dC or by DM in a time-dependent manner (Figure 7B).

We next examined the methylation status of Myog451 in vivo. As seen in Figure $7 \mathrm{C}$, bisulfite mapping using genomic DNA from adult tissues showed that Myog451 was highly methylated in non-muscle samples (brain, $62.5 \%$; kidney, $68.5 \%$ ), but less so in skeletal muscle (39.2\%). The methylation level of Myog451 in the limb
A

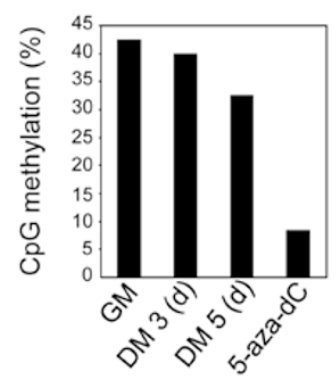

C

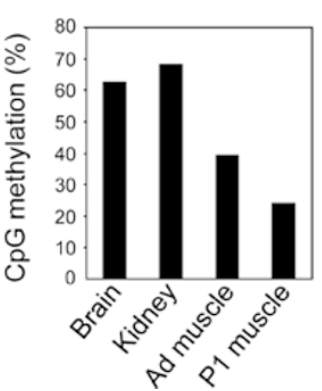

B
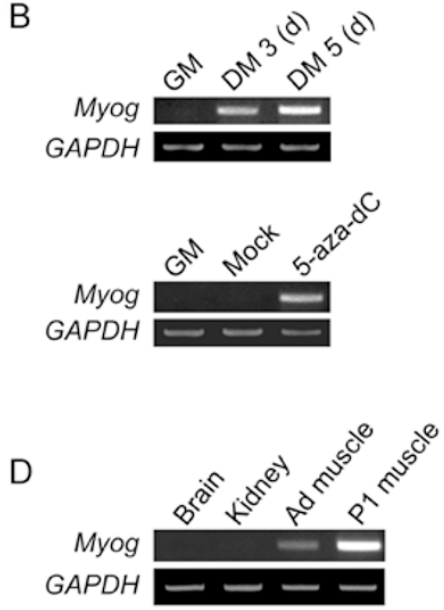

Figure 7 The methylation status of the 451-bp proximal Myog promoter is inversely correlated with Myog expression. (A, C) Genomic DNA was extracted and assayed by bisulfite sequencing analysis, and overall cytosine methylation percentages for Myog451 are shown in the black bars. The percentage values indicate the total methylation level of the $12 \mathrm{CpG}$ sites in Myog451; methylation of each CpG dinucleotide was assessed for 10 individual clones. (A) Methylation status of Myog451 in C2C12 cells under growth medium (GM), DM treatment for 3 or 5 days, and $1 \mu \mathrm{M} 5$-aza-dC treatment for 3 days. (B) Semiquantitative RT-PCR analysis shows the expression of Myog in DM-treated $\mathrm{C} 2 \mathrm{C} 12$ cells at the time points indicated (upper panel) or in cells treated with $1 \mu \mathrm{M} 5$-aza-dC for 3 days (lower panel). Mock, same volume of DMSO. (C) Methylation status of Myog451 in 2-month-old mouse tissues (brain, kidney and skeletal limb muscle (Ad muscle)) and postnatal day 1 limb muscle (P1 muscle). (D) Semiquantitative RT-PCR for Myog expression in the indicated 2-month-old mouse tissues and in postnatal day 1 limb muscle (P1 muscle). GAPDH served as a loading control for $\mathbf{B}$ and $\mathbf{D}$. of postnatal day 1 (P1) mice was even lower (24.2\%). In particular, demethylation at $-340,-308,-230,-123$, $-75,-10$ and $+3 \mathrm{CpG}$ sites correlated with the up-regulation of Myog mRNA expression in adult muscles as compared with non-muscle tissues (Supplementary information, Figure S5D-S5E, Figure 7D). Notably, $-340,-308$, $-230,-75$ and $-10 \mathrm{CpG}$ sites were more demethylated during skeletal muscle development (Figure 7D). These data imply that demethylation at specific $\mathrm{CpG}$ sites may be responsible, in part, for Myog transcription in vivo.

siRNA-mediated knockdown of CIBZ does not significantly affect the methylation status of Myog451

We next asked whether CIBZ abrogation affected the methylation status of Myo451. To address this, genomic DNA extracted from CIBZ siRNA-transfected C2C12 cells (described in Figure 2) was analyzed by SBS. As shown in Supplementary information, Figure S7, siRNAmediated CIBZ knockdown in $\mathrm{C} 2 \mathrm{C} 12$ cells did not greatly influence $\mathrm{CpG}$ methylation, either overall (Supplementary information, Figure S7A) or at individual sites (Supplementary information, Figure S7B) in Myog451. These data indicate that induction of Myog transcription by CIBZ knockdown is independent of demethylation of CpG sites in Myog451.

\section{Discussion}

We previously reported that CIBZ plays an important role in the negative regulation of apoptosis in murine cells [21]. Interestingly, genome-wide association data demonstrate that the gene most frequently associated with human stature is ZBTB38 [29], although the reasons behind this are unclear. We show here that CIBZ is down-regulated both in differentiating $\mathrm{C} 2 \mathrm{C} 12$ cells and in regenerating skeletal muscle. Consistent with this, gain- and loss-of-function data indicate that CIBZ suppresses myogenic differentiation in cultured cells. Our data strongly suggest that these effects are attributable, at least in part, to CIBZ suppressing its direct target, Myog. We also reveal that demethylation in and around the Myog promoter region facilitates Myog transcription in vitro and in vivo.

DNA methylation is hypothesized to act as an on-off switch for developmentally regulated and/or tissue-specific gene expression. Two current models are considered to account for the transcriptional repression of a methylated gene, and it is likely that both are biologically relevant $[13,30]$. The first is that methylation of $\mathrm{CpG}$ dinucelotides impairs the binding of some transcriptional factors to their cognate DNA sequences. The second is that methylated cytosines at $\mathrm{CpG}$ dinucleotides recruit 
Table 1 Summary of methylation level of Myog451, CIBZ protein expression, and Myog transcription

\begin{tabular}{lll}
\hline C2C12 cells & CIBZ expression & Myog transcription \\
\hline GM & High & Low \\
DM-early stage & Very low & Weak \\
DM-late stage & High $^{\text {a }}$ & Very strong \\
5-aza-dC & Moderate $\sim$ High & Off \\
Non-muscle tissues & Very high & Moderate \\
Pdult skeletal muscle & Weak & Very strong \\
\hline
\end{tabular}

The black portions of the pie chart represent the proportion of all $\mathrm{CpG}$ dinucleotides in Myo451.

${ }^{a}$ Indicates that highly expressed CIBZ cannot bind to demethylated Myog451.

MBPs that specifically recognize these sites. Our data suggest that the latter model is valid (see Table 1). In proliferating $\mathrm{C} 2 \mathrm{C} 12$ myoblasts, where Myog451 is moderately methylated and CIBZ is highly expressed, Myog transcription is turned off (Figures 1A, 7A and 7B). In the early stage of differentiation following DM induction, down-regulation of CIBZ protein, together with the demethylation of several CpG sites in Myog451, correlates with induction of Myog transcription (Figures 1A, 7A and 7B, Supplementary information, Figure S5C). In the late stage of differentiation, progressive hypomethylation of Myog451 and a profound decrease of CIBZ protein correlate with further up-regulation of $M y o g$ (Figures 1A, 7A and 7B, Supplementary information, Figure S5C). Upon treatment of $\mathrm{C} 2 \mathrm{C} 12$ cells with 5-aza$\mathrm{dC}$, the almost complete demethylation of Myog 451 coincides with elevated expression of Myog (Figure 7A and 7B, Supplementary information, Figure S5C); ChIP data indicated that CIBZ could not bind to demethylated Myog451 (Figure 4C). Notably, the induction of Myog transcription by CIBZ knockdown is independent of demethylation of $\mathrm{CpG}$ sites in Myog451 (Supplementary information, Figure S7). Taken together, these data suggest that either demethylation of $\mathrm{CpG}$ sites in Myog451 or CIBZ down-regulation leads to the induction of Myog transcription, and that these two events together result in a further up-regulation of $M y o g$, contributing to the terminal differentiation of $\mathrm{C} 2 \mathrm{C} 12$ myoblasts.

We previously showed that CIBZ is ubiquitously expressed in adult mouse tissues [20]; its expression is high in kidney, and especially so in skeletal muscle (Supplementary information, Figure S6). In non-muscle tissues, such as brain and kidney, hypermethylation of Myog451 correlates with the silencing of Myog (Figure 7C and 7D). Conversely, partial demethylation of Myog451 correlates with the induction of Myog in adult skeletal muscle (Figure 7C and 7D). Moreover, the dramatic de- crease in CIBZ protein level and the demethylation of Myog451 coincide with the remarkable up-regulation of Myog in postnatal day 1 muscle relative to adult muscle (Figure 7C and 7D, Supplementary information, Figure S6). These data suggest that CIBZ protein participates in the regulation of postnatal growth of skeletal muscle. The precise reason for CIBZ protein degradation during skeletal muscle growth and regeneration remains unclear. The proteasome inhibitor MG132 can efficiently prevent CIBZ degradation during myogenic differentiation in $\mathrm{C} 2 \mathrm{C} 12$ cells [21], suggesting that the ubiquitinproteasome pathway is involved in the regulation of CIBZ protein in vivo. Collectively, these observations lead us to conclude that the silencing of the Myog locus is coordinately regulated by the levels of CIBZ expression and of Myog451 CpG methylation in tissues, and during postnatal growth of skeletal muscle.

Although the Myog promoter is $\mathrm{CpG}$ island-free, multiple $\mathrm{CpG}$ dinucleotides occur within and in close proximity to this promoter (Figure 5A). During revision of this manuscript, Steffens et al. reported that exposure of $\mathrm{C} 2 \mathrm{C} 12$ cells to sodium arsenite delayed DM-induced differentiation [31]. Increased methylation of the same $12 \mathrm{CpG}$ sites at the Myog promoter that we describe here correlated with a reduction in Myog transcription, which may consequently lead to a delay in myogenic differentiation [31]. Generally, the number of methylated CpGs and the methylation levels of $\mathrm{CpG}$ sites appear to coordinately control gene transcription in cells and tissues, although the molecular mechanism underlying this phenomenon remains poorly understood $[13,30]$. We observed that the 12 CpGs in Myog451 are important to mediate methylation-dependent silencing of $\mathrm{Myog}$ (Figure 6C), and that demethylation of these sites correlates with transcriptional activation of Myog (Figure $7 B$, Supplementary information, Figure S5B-5C). Since some specific $\mathrm{CpG}$ sites are more demethylated during 
C2C12 myoblast differentiation (Supplementary information, Figure S5C), as well as in adult mouse tissues and in muscle development (Supplementary information, Figure S5E), we suppose that, under various circumstances, these $\mathrm{CpG}$ sites might collectively be critical for the regulation of Myog transcription.

Currently, two major families of MBPs are known in vertebrates: methyl-CpG-binding domain (MBD) proteins and $\mathrm{C}_{2} \mathrm{H}_{2}$ zinc finger proteins, all of which bind preferentially to methylated $\mathrm{CpG}$ and repress transcription. The MBD-containing proteins include MBD1, MBD2, MBD4 and MeCP2 [32, 33]; Kaiso, ZBTB4 and ZBTB38 (CIBZ in mouse) use zinc fingers to bind methylated CpG [34]. Compared to the loss of DNA methyltransferases, the consequence of loss of MBDcontaining proteins in animals is quite mild, with the exception of Kaiso depletion in Xenopus laevis, where xKaiso is linked with the maintenance of transcriptional silencing before the onset of zygotic transcription [35]. Interestingly, MBD1, Kaiso and ZBTB4 have the ability to bind, albeit weakly, unmethylated DNA: MBD1 binds unmethylated $\mathrm{CpG}$ through its $\mathrm{CXXC} 3$ motif, while Kaiso and ZBTB4 recognize CTGCNA sites via zinc fingers $[19,36]$. These findings raise questions of whether these MBPs bind preferentially to a particular site (methylated or non-methylated), and of what the biological significance is of such binding. By analyzing Kaiso-binding sites in HEK293T cells, Ruzov et al. found that Kaiso associates with methylated CpGs but not CTGCNA sites, and importantly, their data show that only the methylated DNA-binding function of Kaiso is required in early Xenopus laevis development [37]. It should be noted that binding of MBPs to methylated DNA appears to be sequence-specific: additional DNA sequence surrounding the $\mathrm{CpG}$ site(s) is important [38, 39].

So far, no MBP has been reported to directly regulate the transcription of Myog. Among the MBD-containing MBPs, the expression of MBD2 and MeCP2 is very low in proliferating myoblasts but increases during myogenic differentiation [40], suggesting that their main role is in terminal muscle differentiation. In support of this hypothesis, up-regulation of these two proteins leads to the reorganization of heterochromatin, and contributes to the terminal differentiation of myotubes [40]. Upon oxidative stress induction, the nuclear translocation of focal adhesion kinase and its consequent interaction with MBD2 have been reported to induce skeletal muscle differentiation [41]. However, it remains unclear whether MBD2 acts alone to influence early myogenic differentiation, or regulates $M y o g$ transcription directly. Of the $\mathrm{C}_{2} \mathrm{H}_{2}$ zinc finger MBPs, CIBZ and ZBTB4 can bind to a single methylated $\mathrm{CpG}$, whereas Kaiso only recognizes
DNA sequences that contain at least two adjacent methyl-CpGs [19, 42]. Since Myog451 contains 12 CpG sites but lacks two consecutive CpGs, Kaiso probably cannot bind to the dispersed methylated CpGs in Myog451. Most recently, artificial selection of ZBTB4 binding sites in vitro demonstrated that ZBTB4 binds specifically to methylated ${ }^{\mathrm{C}} / \mathrm{CGC}_{\mathrm{A}} /{ }_{\mathrm{T}} \mathrm{AT}$, a sequence to which Kaiso and ZBTB38 can also bind [39]. The absence of such a consensus sequence in Myog451 suggests that ZBTB4 does not bind to Myog451. Further studies are required to determine whether other MBPs, including MBD1 and MBD4, contribute to early myogenic differentiation, and directly regulate Myog transcription through interactions with the Myog451 region, both in cells and in tissues.

In conclusion, our data demonstrate that CIBZ negatively regulates $\mathrm{C} 2 \mathrm{C} 12$ myoblast differentiation. This finding leads us to hypothesize that CIBZ may also affect satellite cell differentiation in vivo, one of the crucial steps of skeletal muscle regeneration. The observation that CIBZ down-regulation may be required for skeletal muscle regeneration in mice (Figure 1B) suggests that suppression of CIBZ would promote muscle regeneration in myodegenerative diseases. Drugs that antagonize CIBZ may offer opportunities for the treatment of skeletal muscle disorders.

\section{Materials and Methods}

\section{Cell culture, DM and 5-aza-dC treatment}

C2C12 and 293T cells were cultured in DMEM supplemented with $15 \%$ or $10 \%$ fetal bovine serum for C2C12 cells and $293 \mathrm{~T}$ cells, respectively, as described previously [21]. To induce myogenic differentiation of $\mathrm{C} 2 \mathrm{C} 12$ cells, subconfluent cells were cultured with DM consisting of DMEM supplemented with $2 \%$ horse serum. Demethylation studies were performed with 5-aza2'-deoxycytidine (5-aza-dC) (Sigma-Aldrich) at a concentration of 0.5-1 $\mu \mathrm{M}$. Cells treated with equivalent amounts of DMSO served as a negative control. The medium for cells cultured with 5 -aza-dC or DMSO was replaced every $24 \mathrm{~h}$. Cells were harvested at different time points after 5 -aza-dC treatment.

\section{Semiquantitative RT-PCR}

Semiquantitative RT-PCR was conducted as described previously [21]. The primers designed were confirmed as unique in the nonredundant NCBI database. Primers for the mouse genes are shown in Supplementary information, Data S1. The annealing temperature was $58{ }^{\circ} \mathrm{C}$ for reactions using all primer sets. Reaction products were separated in a $2 \%$ agarose gel and visualized by ethidium bromide staining. To confirm the identity of PCR products, single bands of the expected size were excised from the gels and sequenced. GAPDH mRNA expression served as an internal control.

\section{siRNA and transient transfection}

C2C12 cells were transfected with $1 \mathrm{nM} \mathrm{CIBZ-specific} \mathrm{Dicer}$ substrate siRNA duplexes, or with DS scrambled negative control 
(Integrated DNA Technologies), as described previously [21]. Transfection with siRNA was carried out with INTERFERin reagent (Polyplus Transfection) according to the manufacturer's instructions. The siRNA-transfected cells were used 24-48 h after transfection for RT-PCR and western blotting. Transient transfections of $\mathrm{C} 2 \mathrm{C} 12$ cells with CIBZ constructs were described previously [20]. Briefly, cells were transfected with Myc-tagged CIBZ and its deletion mutant using Lipofectamine 2000 (Invitrogen) according to the manufacturer's instructions.

Reporter constructs, in vitro methylation and luciferase assay

CpG-free vector pCpGL was a kind gift from Dr Michael Rehli [28]. For the pCpGL-Myog451 construct, the Myog451 (-406 to $+45 \mathrm{bp}$ ) fragment was generated by PCR with primers (forward: 5'-CCAGGGATCCCTTGTTCCCTTCCTGCCCTGTC-3'; reverse: 5'-CCCAAGCTTGGTCGGAAAAGGCTTGTTCCTG$\left.3^{\prime}\right)$ that incorporated restriction sites (BamHI and HindIII), and subsequently ligated into pre-digested pCpGL vector. DNA sequences were verified using BigDye terminator and an automated sequencer (ABI PRISM310). pCpGL and pCpGL-Myog451 were methylated in vitro by M.SssI (New England Biolabs) according to the manufacturer's recommendations, or mock-methylated in the absence of M.SssI. The extent of pCpGL-Myog451 methylation was verified by digesting the methylated $\mathrm{CpG}$ with HpaII. Transfections were carried out with Lipofectamine 2000 and luciferase assays were performed using a previously described protocol [43]. The thymidine kinase promoter-Renilla luciferase reporter plasmid pRL-TK was used as an internal control. All experiments were performed in triplicate, and the data shown are representative of at least three independent experiments with similar results.

\section{Electrophoretic mobility gel shift assay (EMSA)}

GST-CIBZ ZF1-5 was constructed and purified as described previously [21]. Oligonucleotides used for probes covering the $12 \mathrm{CpG}$ sites were purchased from Greiner bio-one (Tokyo, Japan). Oligonucleotide sequences are shown in Supplementary information, Data S1 about primers. EMSA was performed with digoxigenin-11-dUTP (DIG)-labeled probes using a DIG gel shift kit (Roche) according to the manufacturer's instructions. Sensestrand oligonucleotides were annealed with their complementary strands and 3'-end-labeled with DIG by terminal transferase. The binding reaction was performed with 20 fmol of DIG-labeled probes incubated with GST-CIBZ ZF1-5 in binding buffer containing $50 \mathrm{ng}$ of poly $(\mathrm{dI}-\mathrm{dC})$, in a final volume of $10 \mu \mathrm{l}$, on ice for 20 min. The DNA-protein complex was resolved in a $6 \%$ polyacrylamide gel, and then electroblotted onto a Nylon membrane and UV-crosslinked. The blot was then blocked and incubated with anti-DIG antibody conjugated with alkaline phosphatase at room temperature for $30 \mathrm{~min}$, washed, subjected to chemiluminescent detection with CSPD (disodium 3-(4-methoxyspiro \{1,2-dioxetane$3,2^{\prime}$-(5'-chloro)tricyclo[3.3.1.1 $\left.1^{3,7}\right]$ decan $\}-4$-yl)phenyl phosphate) as the substrate, and exposed to an X-ray film. CpG methylation of the probe was catalyzed by M.SssI as described above. For competition assays, a 150-fold excess of unlabeled probe was preincubated with the binding mixture.

\section{CIBZ antibody production and western blotting}

A new CIBZ polyclonal antibody (C2) was produced by JBios
(Saitama, Japan). Briefly, a KLH-conjugated peptide corresponding to amino acids 1184-1197 (EQKDDIKAFAENVL) of mouse CIBZ was synthesized. Two rabbits were immunized with this peptide according to standard protocols. Blood taken at two and three months after immunization was pooled, and the antibodies were affinity-purified. The specificity of $\mathrm{C} 2$ was confirmed by immunoblotting and comparison with an anti-CIBZ antibody described previously [20] (data not shown). C2 specifically immunoprecipitated endogenous CIBZ from $\mathrm{C} 2 \mathrm{C} 12$ cells (data not shown). Western blotting was performed as described previously [20]. Briefly, cells were sonicated and lysed in lysis buffer. Proteins were separated by $8-15 \%$ SDS-PAGE, transferred to PVDF membranes and probed with the following antibodies: anti-CIBZ (C2), anti-MyoD (C-20, sc-304, Santa Cruz Biotechnology), antiMyog (5FD, sc-52903, Santa Cruz Biotechnology), anti- $\alpha$-tubulin (clone DM 1A, Sigma) and anti-MHC (clone MF20, Developmental Studies Hybridoma Bank). HRP-conjugated anti-mouse or antirabbit IgG (GE Healthcare) was used as the secondary antibody.

\section{ChIP assay}

A ChIP assay was carried out with an EZ-ChIP kit (Millipore) according to the manufacturer's instructions. C2C12 cells were grown to subconfluence on $10-\mathrm{cm}$ plates. After crosslinking for 30 min with $1 \%$ formaldehyde, glycine was added to a final concentration of $0.125 \mathrm{M}$ and the cells were washed 3 times with ice-cold phosphate-buffered saline (PBS) and harvested with SDS-lysis buffer. The chromatin lysate was sonicated on ice to an average DNA length of $500 \mathrm{bp}$. After preclearing the lysates with Protein G Sepharose beads, anti-CIBZ antibody (C2) was used to immunoprecipitate protein-DNA complexes. Anti-MyoD antibody was used as a positive control, and preimmune IgG and distilled water served as negative controls. Amplification of the immunoprecipitated DNA was achieved using Blend Tag DNA polymerase (Toyobo, Osaka, Japan). Primers shown in Supplementary information, Data S1 were designed to evaluate the promoter region (prom) and distal region (dist). The annealing temperature for both prom and dist primers was $55^{\circ} \mathrm{C}$. Each experiment was repeated four times.

\section{Sodium bisulfite genomic sequencing}

Genomic DNA was isolated from $\mathrm{C} 2 \mathrm{C} 12$ cells and mouse tissues (C57BL/6) with the Gentra Puregene genomic DNA purification kit (Roche) according to the manufacturer's instructions. Bisulfite reactions were performed using an EZ DNA MethylationGold kit (Zymo Research) under conditions that allowed for complete conversion of cytosines, but not 5-methylcytosines, to uracil. The modified DNA was amplified by nested PCR with two sets of primers listed in Supplementary information, Data S1 about primers. PCR primers were designed with MethPrimer software [44], and all primer sequences were devoid of $\mathrm{CpG}$ dinucleotides to avoid biased amplification of methylated alleles. Hot-start PCR was performed at $95{ }^{\circ} \mathrm{C}$ for $5 \mathrm{~min}$, followed by a first-round amplification using 42 cycles of $95{ }^{\circ} \mathrm{C}$ for $30 \mathrm{~s}, 45^{\circ} \mathrm{C}$ for $40 \mathrm{~s}$ and 72 ${ }^{\circ} \mathrm{C}$ for $1 \mathrm{~min}$. The conditions for the second amplification were the same as those for the first round of PCR. The PCR products were electrophoresed in $2 \%$ agarose gels and visualized with ethidium bromide. Bands of the expected sizes were isolated and their DNA was extracted using a Wizard SV Gel and PCR clean-up system (Promega). The purified DNA was cloned into pGEM-T (Promega). Ten clones were picked for each sample, and their DNA 
was extracted and sequenced. CpGviewer [45] was used to determine methylation profiles by comparing the sequence of bisulfiteconverted DNA with that of unmodified DNA.

\section{Muscle regeneration assay}

Mice (C57BL/6, 6-week-old males) were anesthetized by intraperitoneal injection of nembutal using a 27 -gauge needle. To introduce a muscle injury, $50 \mu \mathrm{l}$ of $0.075 \%$ CTX from Naja mossambica mossambica (Sigma, C-9759) in PBS was injected into the right muscle tibialis anterior (TA) using a 27-gauge needle [23]. The left side was injected with the same volume of PBS as a control. Muscles were harvested at 1 and 3 days after injection. Mice were killed by cervical dislocation, and total muscle lysates isolated from the CTX-and PBS-injected TA muscles were analyzed by immunoblotting. Expression of Myog mRNA and protein was used as a positive control to evaluate regeneration efficiency.

\section{Acknowledgments}

We are grateful to Dr Ian Smith (Nara Institute of Science and Technology) for critical reading of the manuscript. We especially thank Dr Manabu Sugai (Kyoto University) for valuable discussions about the work. We thank members of the Kawaichi laboratory for technical advice. We thank Dr Michael Rehli (University Hospital Regensburg) for providing the pCpGL plasmid. This work was supported by a grant-in-aid for Scientific Research (C), and the Global COE program, from Japan's Ministry of Education, Culture, Sports, Science and Technology.

\section{References}

1 Hawke TJ, Garry DJ. Myogenic satellite cells: physiology to molecular biology. J Appl Physiol 2001; 91:534-551.

2 Charge SBP, Rudnicki MA. Cellular and molecular regulation of muscle regeneration. Physiol Rev 2004; 84:209-238.

3 Cardasis CA, Cooper GW. An analysis of nuclear numbers in individual muscle fibers during differentiation and growth: a satellite cell-muscle fiber growth unit. J Exp Zool 1975; 191:347-358.

4 Shi X, Garry DJ. Muscle stem cells in development, regeneration, and disease. Genes Dev 2006; 20:1692-1708.

5 Arnold H, Winter B. Muscle differentiation: more complexity to the network of myogenic regulators. Curr Opin Genet Dev 1998; 8:539-544.

6 Knapp JR, Davie JK, Myer A, et al. Loss of myogenin in postnatal life leads to normal skeletal muscle but reduced body size. Development 2006; 133:601-610.

7 Cheng, TC, Wallace MC, Merlie JP, et al. Separable regulatory elements governing myogenin transcription in mouse embryogenesis. Science 1993; 261:215-218.

8 Edmondson DG, Cheng TC, Cserjesi P, et al. Analysis of the myogenin promoter reveals an indirect pathway for positive autoregulation mediated by the muscle-specific enhancer factor MEF-2. Mol Cell Biol 1992; 12:3665-3677.

9 Heidt AB, Rojas A, Harris IS, et al. Determinants of myogenic specificity within MyoD are required for noncanonical E box binding. Mol Cell Biol 2007; 27:5910-5920.

10 Molkentin JD, Olson EN. Combinatorial control of muscle development by basic helix-loop-helix and MADS-box transcription factors. Proc Natl Acad Sci USA 1996; 93:93669373.

11 Benezra R, Davis RL, Lockshon D, et al. The protein Id: A negative regulator of helix-loop-helix DNA binding proteins. Cell 1990; 61:49-59.

12 Lu J, Webb R, Richardson JA, et al. MyoR: A muscle-restricted basic helix-loop-helix transcription factor that antagonizes the actions of MyoD. Proc Natl Acad Sci USA 1999; 96:552557.

13 Bird A. DNA methylation patterns and epigenetic memory. Genes Dev 2002; 16:6-21.

14 Taylor SM, Jones PA. Multiple new phenotypes induced in and 3T3 cells treated with 5-azacytidine. Cell 1979; 17:771779.

15 Szyf M, Rouleau J, Theberge J, et al. Induction of myogenic differentiation by an expression vector encoding the DNA methyltransferase cDNA sequence in the antisense orientation. J Biol Chem 1992; 267:12831-12836.

16 Scarpa S, Lucarelli M, Palitti F, et al. Simultaneous myogenin expression and overall DNA hypomethylation promote in vitro myoblast differentiation. Cell Growth Differ 1996; 7:10511058.

17 Lucarelli M, Fuso A, Strom R, et al. The dynamics of myogenin site-specific demethylation is strongly correlated with its expression and with muscle differentiation. $J$ Biol Chem 2001; 276:7500-7506.

18 Blais A, Tsikitis M, Acosta-Alvear D, et al. An initial blueprint for myogenic differentiation. Genes Dev 2005; 19:553569.

19 Filion GJP, Zhenilo S, Salozhin S, et al. A family of human zinc finger proteins that bind methylated DNA and repress transcription. Mol Cell Biol 2006; 26:169-181.

20 Sasai N, Matsuda E, Sarashina E, et al. Identification of a novel BTB-zinc finger transcriptional repressor, CIBZ, that interacts with CtBP corepressor. Genes Cells 2005; 10:871885.

21 Oikawa Y, Matsuda E, Nishii T, et al. Down-regulation of CIBZ, a novel substrate of caspase-3, induces apoptosis. $J$ Biol Chem 2008; 283:14242-14247.

22 Soulez M, Rouviere C, Chafey P, et al. Growth and differentiation of $\mathrm{C} 2$ myogenic cells are dependent on serum response factor. Mol Cell Biol 1996; 16:6065-6074.

23 Hirata A, Masuda S, Tamura T, et al. Expression profiling of cytokines and related genes in regenerating skeletal muscle after cardiotoxin injection: a role for osteopontin. Am J Pathol 2003; 163:203-215.

24 Yan Z, Choi S, Liu X, et al. Highly coordinated gene regulation in mouse skeletal muscle regeneration. J Biol Chem 2003; 278:8826-8836.

25 Conte C, Ainaoui N, Delluc-Clavieres A, et al. Fibroblast growth factor 1 induced during myogenesis by a transcription-translation coupling mechanism. Nucleic Acids Res 2009; 37:5267-5278.

26 Berkes CA, Bergstrom DA, Penn BH, et al. Pbx marks genes for activation by $\mathrm{MyoD}$ indicating a role for a homeodomain protein in establishing myogenic potential. Mol Cell 2004; 14:465-477.

27 Kiefer H, Chatail-Hermitte F, Ravassard P, et al. ZENON, a 
novel POZ kruppel-like DNA binding protein associated with differentiation and/or survival of late postmitotic neurons. Mol Cell Biol 2005; 25:1713-1729.

28 Klug M, Rehli M. Functional analysis of promoter CpG methylation using a $\mathrm{CpG}$-free luciferase reporter vector. Epigenetics 2006; 1:127-130.

29 Gudbjartsson DF, Walters GB, Thorleifsson G, et al. Many sequence variants affecting diversity of adult human height. Nat Genet 2008; 40:609-615.

30 Hoelzer K, Shackelton LA, Parrish CR. Presence and role of cytosine methylation in DNA viruses of animals. Nucleic Acids Res 2008; 36:2825-2837.

31 Steffens AA, Hong GM, Brain LJ. Sodium arsenite delays the differentiation of $\mathrm{C} 2 \mathrm{C} 12$ mouse myoblast cells and alters methylation patterns on the transcription factor myogenin. Toxicol Appl Phamacol 2010; 250:154-161.

32 Fatemi M, Wade PA. MBD family proteins: reading the epigenetic code. J Cell Sci 2006; 119:3033-3037.

33 Fischle W. Talk is cheap - cross-talk in establishment, maintenance, and readout of chromatin modifications. Genes Dev 2008; 22:3375-3382.

34 Prokhortchouk E, Defossez PA. The cell biology of DNA methylation in mammals. Biochim Biophys Acta 2008; 1783:2167-2173.

35 Ruzov A, Dunican DS, Prokhortchouk A, et al. Kaiso is a genome-wide repressor of transcription that is essential for amphibian development. Development 2004; 131:6185-6194.

36 Clouaire T, de las Heras JI, Merusi C, Stancheva I. Recruitment of MBD1 to target genes requires sequence-specific interaction of the MBD domain with methylated DNA. Nucleic Acids Res 2010; 38:4620-4634.

37 Ruzov A, Savitskaya E, Hackett JA, et al. The non-methylated DNA-binding function of Kaiso is not required in early Xeno- pus laevis development. Development 2009; 136:729-738.

38 Klose RJ, Sarraf SA, Schmiedeberg L, McDermott SM, Stancheva I, Bird AP. DNA binding selectivity of MeCP2 due to a requirement for $\mathrm{A} / \mathrm{T}$ sequences adjacent to methyl-CpG. Mol Cell 2005; 19:667-678.

39 Sasai N, Nakao M, Defossez PA. Sequence-specific recognition of methylated DNA by human zinc-finger proteins. Nucleic Acids Res 2010; 38:5015-5022.

40 Brero A, Easwaran HP, Nowak D, et al. Methyl CpG-binding proteins induce large-scale chromatin reorganization during terminal differentiation. $J$ Cell Biol 2005; 169:733-743.

41 Luo SW, Zhang C, Zhang B, et al. Regulation of heterochromatin remodelling and myogenin expression during muscle differentiation by FAK interaction with MBD2. EMBO J 2009; 28:2568-2582.

42 Prokhortchouk A, Hendrich B, Jorgensen H, et al. The p120 catenin partner Kaiso is a DNA methylation-dependent transcriptional repressor. Genes Dev 2001; 15:1613-1618.

43 Agata Y, Matsuda E, Shimizu A. Two novel Kruppel-associated box-containing zinc-finger proteins, KRAZ1 and KRAZ2, repress transcription through functional interaction with the corepressor KAP-1 (TIF1beta/KRIP-1). J Biol Chem 1999; 274:16412-16422.

44 Li L, Dahiya R. MethPrimer: designing primers for methylation PCRs. Bioinformatics 2002; 18:1427-1431.

45 Carr IM, Valleley EMA, Cordery SF, et al. Sequence analysis and editing for bisulphite genomic sequencing projects. Nucleic Acids Res 2007; 35:e79.

46 Matsuda E, Agata Y, Sugai M, Katakai T, Gonda H, Shimizu A. Targeting of Krüppel-associated box-containing zinc finger proteins to centromeric heterochromatin. Implication for the gene silencing mechanisms. J Biol Chem 2001; 276:1422214229.

(Supplementary information is linked to the online version of the paper on the Cell Research website.) 